\title{
Neutron wave-interference experiments with adiabatic passage of neutron spin through resonant coils
}

\author{
S. V. Grigoriev, ${ }^{1,2, *}$ R. Kreuger, ${ }^{1}$ W. H. Kraan, ${ }^{1}$ F. M. Mulder, ${ }^{1}$ and M. Th. Rekveldt ${ }^{1}$ \\ ${ }^{1}$ Interfacultair Reactor Instituut, TU-Delft, $2629 \mathrm{JB}$ Delft, The Netherlands \\ ${ }^{2}$ Petersburg Nuclear Physics Institute, Gatchina, St. Petersburg 188350, Russia
}

(Received 21 December 2000; published 7 June 2001)

\begin{abstract}
Neutron resonance spin echo phenomena, produced by resonance coils with adiabatic passage of the neutron spin, are investigated experimentally and theoretically. The adiabatic passage of the neutron spin through a resonance coil requires a specific configuration of the magnetic field. The solution of the Schrödinger equation, obtained for the required configuration, shows the probability of a spin-flip process; it also shows the phase shift, which the neutron experienced, when it flies through it. The precession phase inside the coil consists of three contributions in the rotating frame approach. The first, biggest contribution is the phase of the rotating frame $\omega_{0} \tau$. The second is the precession phase of the neutron spin in the rotating frame since it follows adiabatically the effective field as seen in this frame. The third, smallest contribution is Berry's phase since the magnetic field rotates over an angle approaching $\pi$ in this rotating frame. This rotation is followed adiabatically by the neutron spin. Finally, the amplitude of the interference pattern and the phase shift between the neutron-spin states are derived for a system consisting of two such flippers. The theoretical consideration is experimentally confirmed.
\end{abstract}

DOI: 10.1103/PhysRevA.64.013614

PACS number(s): 03.75.Dg, 42.87.Bg

\section{INTRODUCTION}

Neutron resonance spin echo (NRSE) is becoming a new, important method to determine the energy transfers in neutron beam scattering experiments [1-3]. In this method, as well as in the conventional spin echo (SE) technique [4], the energy transfer or velocity change of neutrons caused by a sample is measured by comparing the amount of precession before and after scattering. The NRSE technique started when Gähler and Golub proposed an alternative (to the conventional method) possibility to produce spin precession in zero field (ZF) between two resonance spin flippers containing rf coils [1-3]. In a number of articles, they investigated the main principles of the ZF precession and carried out experiments showing advantages and possibilities of the new "mode" in SE spectrometry. In particular, they showed that a pair of the rf flippers, operated in resonance $\omega_{0}=\gamma B_{0}$ and separated by a distance $L$, simulates a dc field integral $2 \gamma B_{0} L$, where $\gamma$ is the neutron gyromagnetic ratio. This effect is well understood if Larmor precession is considered as an interference phenomenon between two superposed neutron waves. Indeed, inside the static magnetic-field region, the incoming neutron wave with momentum $\vec{k}$ will become the superposition of spin state waves with a phase shift that is determined by the momentum difference $\vec{k}_{+}$and $\vec{k}_{-}$. Then after spin flip in an rf coil, neutrons leave the static magnetic field and the momentum difference is doubled because of the energy-conservation law. The second flipper, positioned at a distance $L$ from the first one, cancels the difference in momenta and therefore stops the precession.

As was shown in $[5,6]$, the spin-flip probability of the rf

*Email address: grigor@rvv.lnpi.spb.su flipper plays an important role in such experiments. The flipper is a combination of the permanent field $B_{0}$ and, perpendicular to it, field of amplitude $B_{\text {rf }}$ oscillating at frequency $\nu_{\mathrm{rf}}$. Two conditions, bounding the parameters of these magnetic fields, must be fulfilled to have the spin-flip process complete. First, the frequency of the rf field matches the Zeeman energy difference between the two spin eigenstates produced by the permanent field: $2 \pi \nu_{\mathrm{rf}}=\gamma B_{0}$. Second, the strength of the rf field $B_{\text {rf }}$, the length of the flipper $l$, and the neutron velocity (or wavelength) must satisfy the equation $\gamma B_{\mathrm{rf}} l / v=\pi$. Therefore, the full spin flip is realized for one value of the wavelength spectrum. When a partial rather than a complete spin-flip process occurs, this results in the appearance of four neutron waves in the space between the flippers. These waves interfere and each pair of them produces a new distinct interference pattern $[5,6]$.

To avoid a wavelength dependence of the spin-flip probability, at present we use a neutron resonance spin flipper (NRSF) with adiabatic passage of the spin instead of a conventional one, as has been used until now in the NRSE method. NRSFs with adiabatic passage were applied for the first time at the beginning of 1970s [7,8]. The main characteristics and advantages of this flipper are the following. First, the range of high efficiency (spin-flip probability is practically equal to unity) is limited only by some minimum wavelength $\lambda_{\min }$ of the neutron spectrum. Second, this flipper has a relatively high flipping stability with respect to the effect of external magnetic field. Since a spin-flip process with $\rho=1$ occurs for the "white" neutron spectrum in this flipper, the whole spectrum of neutron wavelengths is involved in the precession, in contrast to experiments in which conventional resonance flippers are used.

It is worthwhile to mention that the principle of adiabatic passage of the spin in neutron experiments has full analogy 
in NMR [9]. The adiabatic passage of the neutron spin requires specific changes in configuration of the magnetic fields of the flipper [10]. The permanent field must have a gradient along the neutron path, so that the resonant point $\left(2 \pi \nu_{\mathrm{rf}}=\gamma B_{0}\right)$ occurs approximately at the middle of the flipper. The amplitude of the oscillating field $B_{\text {rf }}$ also is not constant, but increases from the entrance to the middle, has its maximum there, and then decreases toward the exit of the flipper. The question arises of what is the phase of the neutron wave if its spin interacts with such a flipper. One can expect a distortion of this phase, caused by a violation of the resonance condition over the whole neutron path except for a single point in the middle. To clarify this point, consider a neutron flying through such a magnetic configuration in the frame rotating with the frequency $\omega_{0}=2 \pi \nu_{\text {rf }}$. In this frame, one comes to the problem of the spin behavior in a "slowly" rotating field, namely the resultant of the gradient and amplitude of the rf field. If the adiabatic condition is fulfilled (the Larmor frequency of the spin precession in this rotating frame is much higher than the rotation rate of the field that rotates itself), the neutron spin follows the direction of the resultant magnetic field in the rotating frame and therefore becomes reversed with respect to field in the laboratory frame. The question of the spin phase in the magnetic field, which is adiabatically varied, was intensively discussed in relation with Berry's phase [11]. The discussion resulted in understanding that this phase is a sum of two contributions: the dynamical phase caused by Larmor precession and the geometrical phase caused by rotating the field in space, socalled Berry's phase. Several experiments were carried out to demonstrate Berry's phase in neutron spin rotation [12-14]. It was additionally shown that Berry's concept could be generalized and expanded to NMR interferometry [15-17].

Combining achievements of the NMR and neutron spectroscopy, we conclude that the total phase of the neutron wave in the magnetic field configuration of NRSF with adiabatic passage is a sum of three contributions. The first, biggest contribution is the phase of the rotating frame $\omega_{0} \tau$. The second one is the precession phase of the neutron spin in the rotating frame while it follows the effective field of this frame adiabatically. The third, smallest one is Berry's phase, or the geometric phase, since the magnetic field rotates adiabatically in this rotating frame. This "double" rotationrotating the magnetic field in the rotating frame-seems to be rather confusing, although all contributions, distinguished theoretically, can be visualized experimentally. The formulas describing the spin-flip probability of one flipper are derived in Sec. II. We calculate also the phase shift between interfering neutron waves and how it depends on the parameters of the flipper. The phase shift between neutron spin states and amplitude of the interference pattern are also derived for a system of two NRSFs with adiabatic passage as is required for NRSE spectroscopy. It will be shown that the phase difference between interfering neutron waves is relatively insensitive to variations of the static magnetic field but not to variations of the gradient. As was predicted in [18], the stability is additionally provided by the fact that the magnetic field on both sides of the magnets is mirrored in the NRSE experiment and therefore variations of the permanent mag- netic field will be compensated. Section III gives details concerning the setup. The results of the measurements are given in Sec. IV. The experiment confirms the conclusion made in Sec. II. Although we have not measured Berry's phase in our present experiments, its discussion sheds additional light on the problem of adiabatic passage of a spin in the NRSF. We shall also propose and discuss an experiment with a NRSF with adiabatic passage in which Berry's phase can be observed. Finally, it is wise to notice that many basic ideas about phase accumulation due to spin precession, which are addressed in the present paper, are discussed in a new book by Rauch and Werner [19].

\section{NEUTRON RESONANCE SPIN ECHO WITH ADIABATIC SPIN FLIPPERS}

\section{A. Qualitative description}

The principle of the adiabatic NRS flipper is easy to explain in the classical approach of the rotating frame [8]. Such a flipper consists of two mutually perpendicular magnetic fields. The first is a permanent field with a gradient along the $x$ axis and parallel to the $z$ axis. The other one is an oscillating field with frequency $\omega_{0}$ and amplitude $B_{\mathrm{rf}}(x)$ perpendicular to it. The frequency of the oscillating field is chosen such that resonance occurs at some point near the middle of the region where the gradient is present. The particle trajectory is parallel to the $x$ axis.

As proposed in [10], in order to make the system mathematically treatable, we use the model of the so-called sinecosine modulation of the effective field in the frame rotating at rate $\omega_{0}$ around the $z$ axis of the laboratory system. This means that the amplitude of the oscillating field, $B_{\mathrm{rf}}(x)$, distributed along the neutron path $x=[0, l]$, is described by the sine function

$$
B_{\mathrm{rf}}(x)=A \sin \left(\pi \frac{x}{l}\right)
$$

The dependence on $x$ of the permanent field $B(x)$ is described by the cosine function

$$
B(x)=B_{0}+A \cos \left(\pi \frac{x}{l}\right),
$$

where $B_{0}$ is the field in the center $x_{0}=l / 2, l$ is the length of the flipper, and $A$ is the amplitude of the modulation. The second term of this equation will show up as a permanent gradient field added to the homogeneous field $B_{0}$. It will be denoted as "gradient field."

When the resonance condition for the center is fulfilled $\left(\omega_{0}=\gamma_{n} B_{0}\right)$, then neutrons, as seen in the rotating frame, are affected by the sum of the effective fields: the static field along the $z$ axis, reduced by the value $B_{0}$, and the amplitude of the rf field $B_{\mathrm{rf}}$, which also appears static. Figure 1 shows the schematic picture of the magnetic-field configuration in the rotating frame. For a neutron that flies through such con- 


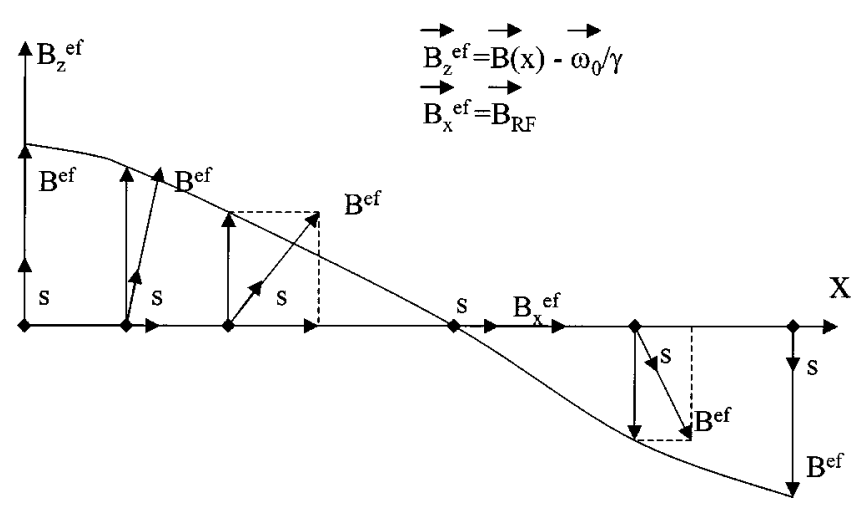

FIG. 1. Scheme of the effective magnetic fields in the frame rotating with frequency $\omega_{0}$. Neutron spin follows the effective magnetic field in this frame, which means a spin flip in the laboratory frame.

figuration with velocity $v$, according to Eqs. (1) and (2), the effective field rotates in the $x-z$ plane with frequency $\Omega$ $=\pi v / l$. At the same time, the neutron spin rotates about this effective field at frequency $\omega_{L}=\gamma A$, where $A$ is the magnitude of the effective field. If the magnitude of the effective field $A$ is large enough, so that $\omega_{L} \gg \Omega$ (the adiabatic approximation), then the neutron spin follows the effective field. Coming back to the laboratory system, this means that spins are reversed on their passage through the flipper, as is shown in Fig. 1.

As is mentioned above, precession of a neutron spin may be effectively produced in the system of two rf spin flippers placed at a distance $L$ from each other. Figure 2(a) shows schematically the system of the magnetic fields of two rf spin flippers with a guide field between them. If spin flipping occurs in the first flipper, the neutron states exchange a photon of energy $\hbar \omega_{0}=-2 \mu_{n} B_{0}$ with the rf field. So during the spin flip in the rf field, neutron spin states split by the magnetic field $B$ with momenta $k_{-}=k_{0}-\left(\mu_{n} B / \hbar v\right)$ and $k_{+}$ $=k_{0}+\left(\mu_{n} B / \hbar v\right)$ will gain or lose an amount of potential energy $\Delta E=2 \mu_{n} B_{0}$. Then, upon leaving the static field, their potential energy is released as a kinetic-energy change. Then the splitting of the wave vector is doubled: $k_{-}$becomes $k_{--}=k_{0}-\left(2 \mu_{n} B_{0} / \hbar v\right), \quad k_{+}$becomes $k_{++}=k_{0}$ $+\left(2 \mu_{n} B_{0} / \hbar v\right)$. This is shown in the $(k, x)$ diagram [Fig. 2(b)], i.e., the diagram of different wave-vector paths between the flippers as a function of position along the beam. In the zero-field region after the flipper, these waves interfere and their phase difference $\phi=\int_{0}^{x}\left[k_{++}\left(x^{\prime}\right)-k_{--}\left(x^{\prime}\right)\right] d x^{\prime}$ $-2 \omega_{0} t$ implies an effective precession in space. This spatial precession, however, takes place in almost zero field [socalled "zero-field" (ZF) precession]. In static experiments, this is unobservable because the phase difference between the two interfering waves continues to grow in time at the rate $2 \omega_{0}$. The time-dependent behavior in $\mathrm{ZF}$ precession may be halted by transmitting the neutron through another $\mathrm{rf}$ spin flipper identical to the first one. Then both $k_{++}$and $k_{--}$ return to $k_{0}$, and also the difference in evolution rate in time $\omega_{\text {rf }}$ disappears by emitting or absorbing the photon in the second flipper. Thus, the growth of both the spatial and time a
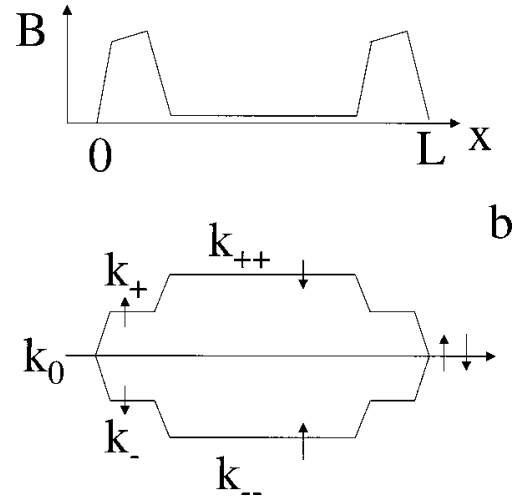

FIG. 2. (a) Sketch of the system of the magnetic fields of the two NR spin flippers with a guide field between them. (b) $(k, x)$ diagram of the wave vectors as a function of position along the beam.

phase difference, i.e., the precession, is halted. The levels $k_{++}$and $k_{--}$represent the splitting in momentum when the spin-flip probability $\rho$ of both flippers is equal to 1 . The amount of $\mathrm{ZF}$ precession (or precession phase) is proportional to the inner area between them and is equal to $\phi$ $=\int\left[k_{++}(x)-k_{--}(x)\right] d x$.

While the precession phase in the space over the length between the flippers is clearly seen, the precession phase obtained by the neutron spin inside NRSF with adiabatic passage should be clarified. Indeed, we might expect that the precession phase would be strongly disturbed and smeared in such a flipper for the following reasons. The static magnetic field has a gradient that results in a distortion of the $k$-level splitting. If the $x$ coordinate of the spin flip has an uncertainty over the length of the flipper, the difference between $k_{+}$and $k_{-}$(therefore the phase difference) might also be smeared. However, the phase will be shown to have a welldefined value in the following quantitative approach. We will conclude that the precession phase inside the flipper is made up of three contributions (in the rotating frame approach): the phase of the rotating frame $\omega_{0} \tau$, the precession phase of the neutron spin in the rotating frame $\int \gamma\left|B_{\text {eff }}(t)\right| d t$, and since it follows adiabatically the effective field, a contribution of the rotation of the effective field over $\pi$ or Berry's phase.

\section{B. Solution of the Schrödinger equation}

In order to derive these conclusions quantitatively, we have to treat the behavior of a plane neutron wave $\Psi\left(t_{0}\right)$ with initial occupation numbers at time $t_{0}\left(\begin{array}{c}\alpha\left(t_{0}\right) \\ \beta\left(t_{0}\right)\end{array}\right)$ in the system of two NRSFs with adiabatic passage, separated in space by the distance $L$ as a solution of the Schrödinger equation. (Here $t_{0}$ is the time at which the neutron enters the first flipper.) First we consider the wave of a neutron with velocity $v$ passing along with coordinate $x$ through the magneticfield configuration, described by Eqs. (1) and (2). The Schrödinger equation for this system can be written as 


$$
i \hbar \frac{d \Psi}{d t}=\left(\begin{array}{ll}
-\mu_{n}\left[B_{0}+A \cos (\pi x / l)\right] & \mu_{n} A \sin (\pi x / l) \exp \left(i \omega_{0} t\right) \\
\mu_{n} A \sin (\pi x / l) \exp \left(-i \omega_{0} t\right) & \mu_{n}\left[B_{0}+A \cos (\pi x / l)\right]
\end{array}\right) \Psi(t) .
$$

Adhering to the standard way of solving the system of coupled differential equations (3), first we move into a coordinate system rotating with frequency $\omega_{0}$ whose $z$ axis coincides with that of the laboratory system. Thus we transform away the exponential or oscillating part of the nondiagonal terms and reduce the diagonal terms by the value $\hbar \omega_{0} / 2 \mu_{n}$. When $\omega_{0}$ satisfies the resonance condition at the center of the flipper,

$$
\omega_{0}=\gamma B_{0}
$$

with $\gamma=-2 \mu_{n} / \hbar$, we reduce the problem to the solution of the following system of equations:

$$
i \hbar \frac{d \Psi_{1}}{d t}=\left(\begin{array}{ll}
-\mu_{n} A \cos (\pi x / l) & \mu_{n} A \sin (\pi x / l) \\
\mu_{n} A \sin (\pi x / l) & \mu_{n} A \cos (\pi x / l)
\end{array}\right) \Psi_{1}(t) .
$$

Writing $x=v t$, Eq. (5) is transformed to the problem of a neutron spin in a magnetic field of amplitude $A$ rotating with frequency $\Omega=\pi v / l$. This problem was extensively discussed recently $[11-13]$ as that concerning the manifestation of Berry's phase in neutron experiments. Indeed, Berry's law has its simplest form in the case of a spin- $\frac{1}{2}$ particle in an external magnetic field. When this magnetic field is varied adiabatically such that the precession frequency of the spin in the field is much higher than the rotating speed of the field itself, then the spin phase depends not only on the interior dynamics, but also on its geometric history [11]. This frequency $\Omega$ will show up in the resulting phase of the neutron spin. How it happens can be easily understood if one transforms into a new rotating frame about the $y$ axis with frequency $\Omega$. To distinguish the two rotating systems that were introduced, we call the system rotating with frequency $\omega_{0}$ frame I, and the system rotating with frequency $\Omega$ frame II. After the second transformation, the neutron spin encounters effectively static magnetic fields. Then we get for the system of equations

$$
i \hbar \frac{d \Psi_{2}}{d t}=\left(\begin{array}{cc}
-\mu_{n} A & i \Omega / 2 \\
-i \Omega / 2 & \mu_{n} A
\end{array}\right) \Psi_{2}(t) .
$$

In this new system (frame II), the rotation of the field reveals itself like a permanent magnetic field of strength $\Omega / \gamma$. This newly formed field makes its own contribution to the spin phase.

The last step, which should be done, is to revolve frame II over the angle $\phi_{0}$ defined by the ratio between value $\gamma A$ and $\Omega$ such that $\cos \phi_{0}=A / \sqrt{A^{2}+(\Omega / \gamma)^{2}}$ and $\sin \phi_{0}$ $=(\Omega / \gamma) / \sqrt{A^{2}+(\Omega / \gamma)^{2}}$. Here $\gamma A$ is, in fact, the frequency of the Larmor precession in frame $\mathrm{I}$, and $\Omega$ is the rotating speed of the magnetic field in frame I. Therefore, we introduce $k$ $=\gamma A / \Omega$ as an adiabaticity parameter of the rotating frame I and rewrite $\cos \phi_{0}=k / \sqrt{k^{2}+1}$ and $\sin \phi_{0}=1 / \sqrt{k^{2}+1}$. After rotating the system through the angle $\phi_{0}$, we have the system of the uncoupled equations with simple solutions:

$$
\Psi_{3}^{1,2}(t)=\Psi_{3}^{1,2}(0) \exp \left(\mp i \sqrt{k^{2}+1} \Omega t / 2\right) .
$$

The wave functions $\Psi, \Psi_{1}, \Psi_{2}$, and $\Psi_{3}$ are related by matrixes of transformations from the laboratory system to frame I, then from frame I to frame II, and from frame II to the system rotated through the angle $\phi_{0}$, respectively. Then after returning to the laboratory system and accounting for the boundary conditions, one gets the solution for a neutron leaving the system at a time $t_{1}+\tau($ where $\tau=l / v)$ :

$$
\Psi\left(t_{1}+\tau\right)=\hat{C}\left(t_{1}, \tau\right) \Psi\left(t_{1}\right),
$$

where $\hat{C}\left(t_{1}, \tau\right)$ is a $2 \times 2$ matrix with elements

$$
\begin{gathered}
C_{11}=C_{22}^{*}=\sin \phi_{0} \sin \phi \exp \left(i \omega_{0} \tau / 2\right), \\
C_{12}=-C_{21}^{*}=-\left[\cos \phi-i \cos \phi_{0} \sin \phi\right] \exp \left(i \omega_{0}\left(t_{1}+\tau / 2\right)\right) .
\end{gathered}
$$

Here we introduced the designation $\phi=\left(\sqrt{k^{2}+1}-1\right) \Omega \tau / 2$. The extra term $-\Omega \tau / 2$ insures $\phi=0$ when there is no field with amplitude $A$.

\section{NRSF with adiabatic passage}

Let us derive the expression for the spin-flip probability $\rho$ from Eqs. (8)-(10). Assuming the initial occupation numbers $\alpha\left(t_{0}\right)=1$ and $\beta\left(t_{0}\right)=0$, the spin-flip probability is given by the occupation number of the spinor component $\downarrow$ after the flipper:

$$
\rho=\beta^{*}\left(t_{1}+\tau\right) \beta\left(t_{1}+\tau\right)=\frac{k^{2}+\cos ^{2} \phi}{k^{2}+1}=1-\frac{\sin ^{2} \phi}{k^{2}+1} .
$$

Hence $\rho$ depends on the value of the parameter $k$, which is the adiabaticity parameter of the NRSF with the gradient field, which is the only remaining field in the rotating frame I and which can be rewritten $k=\gamma A l / \pi v$.

It is seen from Eq. (11) that resonant spin flip occurs for big $k$, i.e.,

$$
k=\gamma l A / \pi v \gg 1 .
$$

The inequality [Eq. (12)] is the adiabatic condition imposed on the length of the flipper $l$, the magnitude of the gradient field $A$, and the value of velocity, $v$. Accounting for the relation between velocity $v$ and wavelength $\lambda[v=\beta / \lambda$, where $\left.\beta=3.958 \times 10^{5}\left(\AA \mathrm{cm} \mathrm{s}^{-1}\right)\right]$, it is immediately seen that at given $l$ and $A$, the condition [Eq. (12)] is well satisfied 
for a wavelength range beyond a certain minimum. Hence the spin-flip probability $\rho$ of NRSF is highly stable and equal practically to unity for a broad wavelength spectrum at $\lambda$ $>\lambda_{\min }$. This last conclusion is the basis for designing the broadband adiabatic spin flipper [10].

The phase shift between interfering neutron waves with opposite spin states inside the "adiabatic" resonant flipper is also derived from Eqs. (8)-(10). For that, we assume the initial occupation numbers $\alpha\left(t_{0}\right)=1 / \sqrt{2}$ and $\beta\left(t_{0}\right)=1 / \sqrt{2}$. Then the polarization components $P_{x}$ and $P_{y}$, perpendicular to the magnetic field, are given by

$$
\begin{aligned}
P_{x}= & \left\langle\sigma_{x}\right\rangle=\cos \left(\omega_{0} \tau\right) \cos 2 \phi \\
& -\operatorname{sgn}(A)\left|\cos \phi_{0}\right| \sin \left(\omega_{0} \tau\right) \sin 2 \phi \\
\approx & \cos \left(\omega_{0} \tau+\operatorname{sgn}(A) 2 \phi\right), \\
P_{y}= & \left\langle\sigma_{y}\right\rangle=\sin \left(\omega_{0} \tau\right) \cos 2 \phi \\
& +\operatorname{sgn}(A)\left|\cos \phi_{0}\right| \cos \left(\omega_{0} \tau\right) \sin 2 \phi \\
\approx & \sin \left(\omega_{0} \tau+\operatorname{sgn}(A) 2 \phi\right),
\end{aligned}
$$

because in the adiabatic approximation Eq. (12) one has $\left|\cos \phi_{0}\right|=k / \sqrt{k^{2}+1} \approx\left(1-1 / 2 k^{2}\right) \approx 1$. So the sign of the gradient $\left(\cos \phi_{0} \sim k \sim A\right)$ comes into the phase obtained by the neutron in the flipper. This phase, as seen in Eqs. (13) and (14), is combined from the phase of the rotating frame I $\left(\omega_{0} \tau\right)$, as it is in a conventional flipper [5], and from the phase $(\phi)$, which is in fact the phase of the spin in the magnetic field of the rotating frame I, $\phi=\left(\sqrt{k^{2}+1}\right.$ -1) $\Omega \tau / 2$. In the adiabatic approximation Eq. (12), we can rewrite $\phi \approx \gamma A \tau / 2-(1-1 / 2 k) \Omega \tau / 2$. Here the first term is the dynamical or Larmor phase in the rotating frame I, and the second term is the geometrical or Berry's phase. The last term is much smaller than the former one and it has a rather fundamental interest. Let us neglect it for simplicity.

Considering the question of the phase stability with respect to external influences, which is important for practical applications, first of all we shall admit that the first term $\omega_{0} \tau$ of the total phase depends on the stability of the generator feeding the rf coil, which is always very high. Concerning the phase $\phi$, when the parameter $k$ is varied, although $k \gg 1$, this results in significant variations of the Larmor part of the phase, while Berry's phase has a weak dependence on $k$. Therefore, the distortion of the effective field in the rotating frame I determines the stability of the total phase. When the effective magnetic field is disturbed, the solution, nevertheless, is still valid as long as the adiabatic condition is satisfied. We should only correct for the change of the phase $\phi$. The simplest examples of a distortion of $\overrightarrow{B_{\text {eff }}}$ (which are the point of our interest) are that the permanent field $B_{0}$ changes from the value $\omega_{0} / \gamma$ or the value of the gradient $2 A$ differs from the value of the amplitude $B_{\text {rf }}$ at its maximum, or both together. Nevertheless, under the adiabatic approximation, we can rewrite the phase $\phi$ in the following way:

$$
\phi \approx \frac{\gamma}{2} \int\left|B_{\mathrm{eff}}(t)\right| d t=\frac{\gamma}{2} \int \sqrt{B_{z, \mathrm{eff}}^{2}(t)+B_{x, \mathrm{eff}}^{2}(t)} d t,
$$

where $B_{z, \text { eff }}(t)=B_{0}-\omega_{0} / \gamma+A^{\prime} \cos (\pi v / l) t$ and $B_{x, \text { eff }}(t)$ $=A^{\prime \prime} \sin (\pi v / l) t$. $A^{\prime}$ and $A^{\prime \prime}$ are the amplitudes of the gradient field and the oscillating field as set by the experimentalist. For arbitrary values of $B_{0}, A^{\prime}$, and $A^{\prime \prime}$, the exact solution does not exist [9]. Nevertheless, Eq. (15) can be used in a computer simulation so that we can compare the experimental data of $\phi$ with the calculated data. Furthermore, we notice that a relatively large change of $B_{0}$ results in a small change of the phase $\phi$, while a change in the gradient is directly proportional to the value of the phase $\phi$.

\section{NRSE: Final expressions}

Next the neutrons fly through a space $L$ free of magnetic field. In this space, they are not influenced by a magnetic field until they enter the second flipper at the moment $t_{1}$ $+\tau+T$, where $T=L / v$. So only the time-dependent part of the spinor function changes, i.e., $\Psi\left(t_{1}+\tau\right)$ becomes $\Psi\left(t_{1}\right.$ $+\tau+T)$. Finally, upon leaving the second flipper at $t_{1}+\tau$ $+T+\tau$, the neutron states may be described according to Eq. (5) by $\Psi\left(t_{1}+\tau+T+\tau\right)=\hat{C}\left(t_{1}+\tau+T, \tau\right) \Psi\left(t_{1}+\tau+T\right)$.

Now we proceed to calculate the final polarization in the $x, y, z$ directions using the resulting function $\Psi\left(t_{1}+\tau+T\right.$ $+\tau)$. The polarization component $P_{i}$ is found by calculating $\left\langle\sigma_{i}\right\rangle=\Psi^{*}\left(t_{1}+\tau+T+\tau\right) \sigma_{i} \Psi\left(t_{1}+\tau+T+\tau\right)$, where $\sigma_{i}$ is the $(2 \times 2)$ Pauli matrix for that polarization component. To produce results that can be compared with experiments, we need to specify the polarization state of the neutron beam before entering the first flipper. When we start with polarization along the $y$ direction, the occupation numbers $\alpha\left(t_{0}\right)$ and $\beta\left(t_{0}\right)$ of the spinor components are both equal to $1 / \sqrt{2}$.

The final polarization components $P_{x}$ and $P_{y}$ consist of numerous terms. Most of them are oscillating functions of time $t_{1}$. In addition, they are reduced by the factor $\sin ^{n} \phi_{0}$ with $n=1, \ldots, 4$ and $\sin \phi_{0} \approx 0$. Hence they do not contribute to the time-averaged polarization as measured in our experiments. We may therefore average out or neglect these terms. We obtain the following for the polarization components $P_{x}, P_{y}$ :

$$
\begin{aligned}
P_{x}= & \left\langle\sigma_{x}\right\rangle \approx \rho^{2} \cos \left[2 \omega_{0}(T+\tau)+\operatorname{sgn}\left(A_{1}\right) 2 \phi_{1}\right. \\
& \left.-\operatorname{sgn}\left(A_{2}\right) 2 \phi_{2}\right], \\
P_{y}= & \left\langle\sigma_{y}\right\rangle \approx \rho^{2} \sin \left[2 \omega_{0}(T+\tau)+\operatorname{sgn}\left(A_{1}\right) 2 \phi_{1}\right. \\
& \left.-\operatorname{sgn}\left(A_{2}\right) 2 \phi_{2}\right] .
\end{aligned}
$$

In the above formulas, we suppose that the spin-flip probabilities for both flippers $\rho_{1}$ and $\rho_{2}$ are close to 1 ; that is, their adiabatic parameters $k_{1}, k_{2}$ are much bigger than 1 . We suppose that the gradients $A_{1}$ and $A_{2}$ of the permanent fields in the flippers may differ. Here $\operatorname{sgn}\left(A_{1,2}\right)= \pm 1$ depends on the mutual orientation of the spin and the gradient of the permanent field. When flipper 1 and 2 are identical, the phases $\phi_{1}$ and $\phi_{2}$ are compensated, since the spin entering the second flipper is opposite to the spin entering the first one because of the spin flip in the first flipper. Therefore, the phase of the polarization signal after such mutual compensation is $2 \omega_{0}(T+\tau)$. 


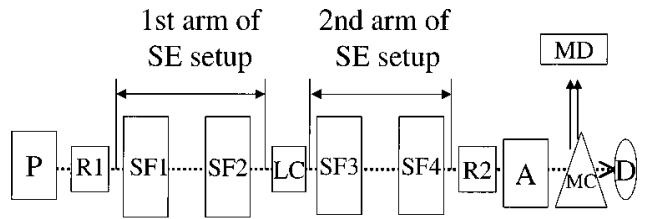

FIG. 3. Schematic drawing of the setup realized at the SP beam line at IRI Delft: $P$ polarizer; $R 1, R 2$ polarization rotators; SF1, $\mathrm{SF} 2, \mathrm{SF} 3$, and SF4 are the NR spin flippers; LC, Larmor coil; $A$, analyzer; $\mathrm{MC}$, monochromator crystal; $\mathrm{MD}$, detector for quasimonochromatic beam; $D$, detector for white beam. The system consisting of SF1 and SF2 makes up the first arm of a spin echo setup while SF3 and SF4 make up its second arm. The phase $\phi$ caused by the difference between first and second arms is measured as the field $B_{1}$ generated in LC needed to compensate this phase.

When both arms of the spin echo setup are operating, Eqs. (16) and (17) become

$$
\begin{gathered}
P_{x}=\rho^{4} \cos [\Delta \Phi], \quad P_{y}=\rho^{4} \sin [\Delta \Phi], \\
\Delta \Phi=2 \omega_{0}\left(T_{1}-T_{2}\right)+\operatorname{sgn}\left(A_{1}\right) 2 \phi_{1}-\operatorname{sgn}\left(A_{2}\right) 2 \phi_{2} \\
-\operatorname{sgn}\left(A_{3}\right) 2 \phi_{3}+\operatorname{sgn}\left(A_{4}\right) 2 \phi_{4} .
\end{gathered}
$$

Here the phase shift $\Delta \Phi$ is determined by the difference in the lengths of the arms $\left(T_{1}\right.$ and $\left.T_{2}\right)$ and by combinations of the phases $\phi_{1,2,3,4}$ with signs determined by mutual orientations of the spin and the magnetic-field gradients of each flipper. From Eqs. (18) and (19), one can see that for $k_{1,2,3,4} \gg 1$ the spin-flip probability does not depend on the relative variations of the permanent magnetic field $B_{0}$ and $A$, and is equal to 1 . So the amplitude of the SE signal has only a little change when the magnetic field $B_{0}$ is varied. The phase shift is changed by varying the values of $A_{1,2,3,4}$ only.

\section{LAYOUT OF THE NRSE EXPERIMENT}

The measurements described in this paper were carried out using the polarizing mirror setup SP at IRI, Delft. Figure 3 gives a schematic outline of this setup. A polychromatic neutron beam emerging from a 2-MW swimming-pool-type reactor is polarized by polarizer $P$. Using rotator $(R 1)$, the polarization of the beam is rotated towards the $y$ axis, i.e., perpendicular to the guide field. The system consisting of two rf spin flippers is located downstream from this rotator $R 1$. This system can be considered as the first arm of a spin echo setup. In our experiments, we set $B_{0}$ equal to $365 \mathrm{G}$ or $760 \mathrm{G}$ and the frequency of the oscillating field equal to 1.08 $\mathrm{MHz}$ or $2.25 \mathrm{MHz}$, respectively, to fulfill the resonance condition Eq. (4) at the center of the flipper. The length of the rf coils is $0.1 \mathrm{~m}$ and they are placed at a center-to-center distance of $L=0.5 \mathrm{~m}$. The permanent magnetic field is produced by an electromagnet with pole shoes such that the magnetic field is relatively homogeneous over the length of the flipper. It drops exponentially outside both sides of the magnet. Two specially constructed coils were placed between the pole shoes to create the gradient of the permanent field. These coils were wound in such a way that the magnetic field is positive over one-half of the magnet and nega- tive over the other half, having zero field at the middle. The current passing through these coils is proportional to the magnitude of the magnetic field ( $z$ component) in the rotating frame I, denoted $A$ in Sec. II. The rf coil with a cross section of $30 \times 30 \mathrm{~mm}^{2}$ is located in the center between these two coils, with the oscillating field along the $x$ axis. With this construction, we could vary the following parameters of the flippers independently: the magnetic field $B_{0}$ from 0 to $1000 \mathrm{G}$, the gradient $2 A$ of the permanent field from 0 to $100 \mathrm{G}$, and the amplitude of the oscillating field from 0 to $40 \mathrm{G}$. After transmission through the two rf flippers, neutrons pass the field stepper and a block-shaped coil [called "Larmor coil" (LC)] of length $0.32 \mathrm{~m}$, which produces a static magnetic field $\vec{B}_{1}$. The second arm consists of two other identical flippers. Their static magnetic field $\vec{B}$ is opposite to the static magnetic field in the first two flippers. The second arm of the SE setup is followed by a second polarization rotator $(R 2)$ and an analyzer $(A)$. By Bragg reflection at a monochromator crystal (MC), neutrons with wavelength $\lambda=0.19-0.23 \mathrm{~nm}$ are reflected into various detectors of the multidetector (MD). The spread of the wavelength spectrum in each detector is $0.02 \mathrm{~nm}$, approximately. The neutron beam transmitted through the crystal monochromator with a "white" spectrum (so-called "white beam") is measured by the detector $D$. The polarization was measured as a function of the phase $\phi$ acquired after passing through both SE arms. The latter is varied by the value of the magnetic field $B_{1}$ in the "Larmor Coil."

\section{RESULTS}

\section{A. Spin-flip probability: Experiment}

As was mentioned above, the spin-flip probability $\rho$ is an important quantity that determines the amplitude of the SE signals. It was measured in a separate experiment with only one flipper between polarizer and analyzer as a function of the permanent field $B_{0}$ while the gradient coil was set to a constant value. In this setup, one measures in fact $P_{z}$. From the definition of the polarization, $\rho$ is connected to $P_{z}$ by

$$
\rho=\left(1-P_{z}\right) / 2 .
$$

Figures 4(a) and 4(b) show broad resonance lines for two values of the resonance frequency $\nu=1.08 \mathrm{MHz}\left(B_{0}^{\text {res }}\right.$ $=365 \mathrm{G})$ and $\nu=2.25 \mathrm{MHz}\left(B_{0}^{\mathrm{res}}=760 \mathrm{G}\right)$, respectively. To demonstrate the ability of the flipper to reverse spin equally for any wavelength, the spin-flip probability for the white beam (full line) and for the monochromatic beam with $\lambda=0.232 \mathrm{~nm}$ (squares) are plotted together. It appears that the spin-flip probability for the monochromatic beam coincides with that of the white beam. The width of the resonance lines at half-height $\left(\rho=\frac{1}{2}\right)$ is determined by the magnitude of the gradient $2 A$ of the permanent magnetic field. In our case, the gradient comprises $80 \mathrm{G}$ from minimum to maximum, as can be deduced from the width of the curves at their bottom. The relative asymmetry of the resonance lines, which is more pronounced in the case of $\nu=2.25 \mathrm{MHz}$ [Fig. 4(b)], is connected with the nonsharp boundary of the mag- 

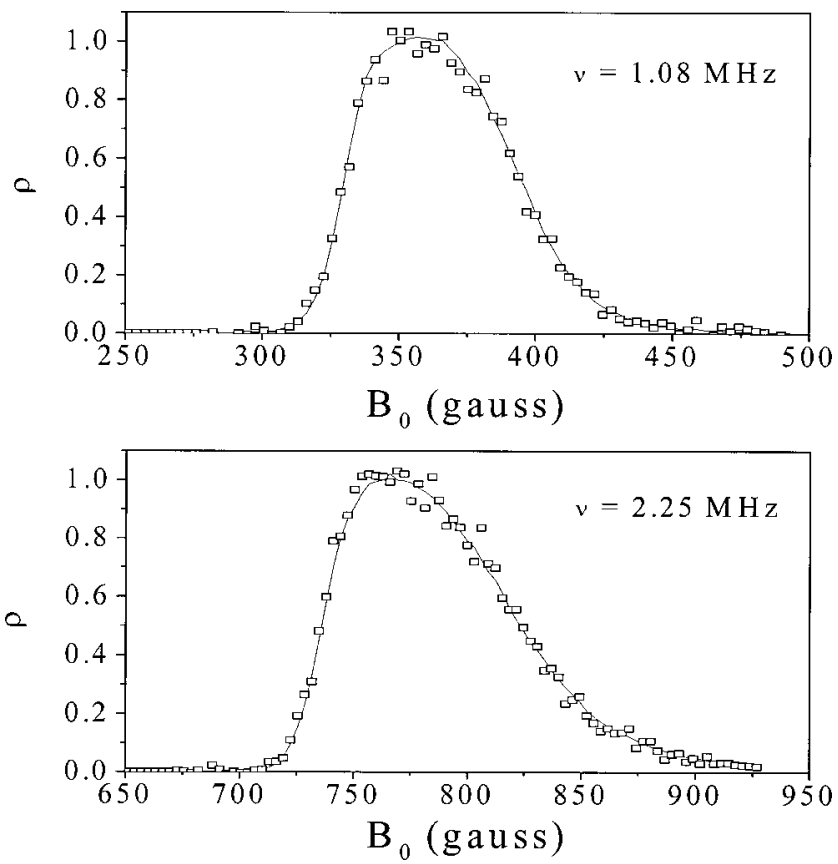

FIG. 4. Dependence of the spin-flip probability $\rho$ in one NR spin flipper on the permanent magnetic field $B_{0}$ for the white spectrum beam (full line) and for the beam with the wavelength $\lambda$ $=0.23 \mathrm{~nm}$ (squares).

netic field $B_{0}$. We point out that the field range with $\rho \approx 1$, a so-called "plateau," extends over a range of about $40 \mathrm{G}$ around $B_{0}^{\text {res }}$, which is about half the field gradient.

Another method to determine $\rho$ is based on using two flippers and measuring the detector count rate for the four different states of the flipper system (both flippers switched off $I_{00}$, one or another flipper on $I_{10}, I_{01}$, both flippers switched on $I_{11}$ ). Using the measured rates $I_{00}, I_{10}, I_{01}, I_{11}$, one can obtain the spin-flip probabilities of both flippers:

$$
\rho_{1}=\frac{1}{2}\left(1+\frac{I_{11}-I_{10}}{I_{00}-I_{01}}\right), \quad \rho_{2}=\frac{1}{2}\left(1+\frac{I_{11}-I_{01}}{I_{00}-I_{10}}\right) .
$$

The efficiency of each flipper, integrated over the whole spectrum, was found for the case of $\nu=1.08 \mathrm{MHz}: \rho_{1}$ $=0.983 \pm 0.003, \rho_{2}=0.997 \pm 0.003, \rho_{3}=0.997 \pm 0.003, \rho_{4}$ $=0.981 \pm 0.003 ;$ and for the case of $\nu=2.25 \mathrm{MHz}$ : $\rho_{1}=0.97 \pm 0.01, \rho_{2}=0.98 \pm 0.01, \rho_{3}=0.99 \pm 0.01, \rho_{4}$ $=0.98 \pm 0.01$. For monochromatic beams with long wavelength, $\rho_{i}$ is even closer to 1 .

The wavelength dependence of the spin-flip probability was also obtained using the so-called Larmor precession spectroscopy [20]. In this method, a polarized neutron beam passes through a magnetic field $B$. Due to Larmor precession, the intensity after the analyzer varies periodically as a function of the field:

$$
I(B)=I_{S}-\int_{0}^{\infty} s(\lambda) P(\lambda) \cos (c \lambda B) d \lambda,
$$

where $I_{S}$ is the average intensity, $s(\lambda)$ is the spectral density, $P(\lambda)$ is the wavelength dependence of the polarizing power, and $c$ is a constant related to the length of the coil producing the field $B$. Fourier transformation of $I(B)$ yields the quantity $F(\lambda)=s(\lambda) P(\lambda)$ :

$$
F(\lambda)=s(\lambda) P(\lambda)=\int_{-B_{\max }}^{B_{\max }}\left[I(B)-I_{S}\right] \cos (c \lambda B) d(c B) .
$$

The intensity $I(B)$ with initial polarization (i.e., rf power in the flippers off) and with polarization after four spin-flip actions (i.e., rf power at $\nu=1.08 \mathrm{MHz}$ on) is presented in Figs. 5(a) and 5(b), respectively. The Fourier transforms of $F_{\text {on }}(\lambda)$ and $F_{\text {off }}(\lambda)$ are plotted in Fig. 5(c). Defining $\bar{\rho}$ as the average of $\rho_{1}, \rho_{2}, \rho_{3}$, and $\rho_{4}$ (i.e., $\bar{\rho}=\sqrt[4]{\rho_{1} \rho_{2} \rho_{3} \rho_{4}}$ ) at any $\lambda$, we find from Eq. (20) that $P_{z}=1-2 \bar{\rho}$. Hence the quotient $F_{\text {on }}(\lambda) / F_{\text {off }}(\lambda)$ is equal to $(1-2 \bar{\rho})^{4}$. From this it follows that $\bar{\rho}=\left(1+\sqrt[4]{F_{\text {on }} / F_{\text {off }}}\right) / 2$. Figures $5(\mathrm{~d})$ and 6 give $\bar{\rho}(\lambda)$ found in this way at $\nu=1.08 \mathrm{MHz}$ and $\nu=2.25 \mathrm{MHz}$, respectively. The wavelength dependence of $\bar{\rho}(\lambda)$ according to Eq. (11) with the sine square averaged function and $k$ $=\gamma A l \lambda /(\pi \beta)$, where $A=40 \mathrm{G}$ and $l=0.1 \mathrm{~m}$, is plotted in the same figures. It can be observed that the spin-flip probability reaches 1 at $\lambda_{\min } \approx 0.2 \mathrm{~nm}$ and remains equal to 1 for the whole wavelength spectrum $\lambda>\lambda_{\min }$ in accordance with the theoretical description.

\section{B. Spin echo experiment}

In the setup described in Sec. III and in accordance with Eqs. (18) and (19), we can vary several parameters to change the phase of the SE signal: two main parameters are the length of the SE arm $T=L / v$ and frequency $\omega_{0}$. To check that the phase, produced by the NRSE, is proportional to the distance $L$ and the resonant frequency $\omega_{0}$, we changed the distance between flippers in the first arm while the second arm stayed without changes for two different frequencies. Figure 7 displays the "white" SE signals for $L$ equal to $50,51,52$, and $53 \mathrm{~cm}$ at $f=1.08 \mathrm{MHz}$ as a function of the phase $\Phi$, i.e., as a function of the compensating field $B_{1}$ in the Larmor coil (LC in Fig. 3). The SE signal, observed from the figures, shifts at a rate $\Delta \Phi_{\exp }=23 \pi$ per $1 \mathrm{~cm}$. The theoretical value of the rate, derived from Eqs. (18) and (19), is $\Delta \Phi_{\text {theor }}=2 \gamma B_{0}^{\text {res }} \bar{\lambda} / \beta=4 \pi \nu \bar{\lambda} / \beta=22 \pi$ per $1 \mathrm{~cm}$ with $\bar{\lambda}$ $=0.2 \mathrm{~nm}$. The rate of change of $\Delta \Phi$ obtained from an analogous experiment at $\nu=2.25 \mathrm{MHz}$ is equal to $\Delta \Phi_{\exp }$ $=42 \pi$ per $1 \mathrm{~cm}$ and from theoretical consideration it is $\Delta \Phi_{\text {theor }}=45 \pi$ per $1 \mathrm{~cm}$. Since $\bar{\lambda}$ is set with an accuracy of about $10 \%$ and the value of $\Delta L$ was measured with the same order of accuracy, the theoretical and measured values of the rate $\Delta \Phi$ coincide within the error bars. The numbers found for $\Delta \Phi$ illustrate the behavior of the biggest contribution of the total phase [Eqs. (18) and (19)], which is proportional to $\omega_{0}$. Thus we have shown experimentally that the NRSE technique works with NRSF with adiabatic passage and the "white" beam may be used in such experiments.

The next two experiments shown below shed light on the key parameters $B_{0}$ and $2 A$ of the flipper, which determine phase stability with respect to variations of the permanent 

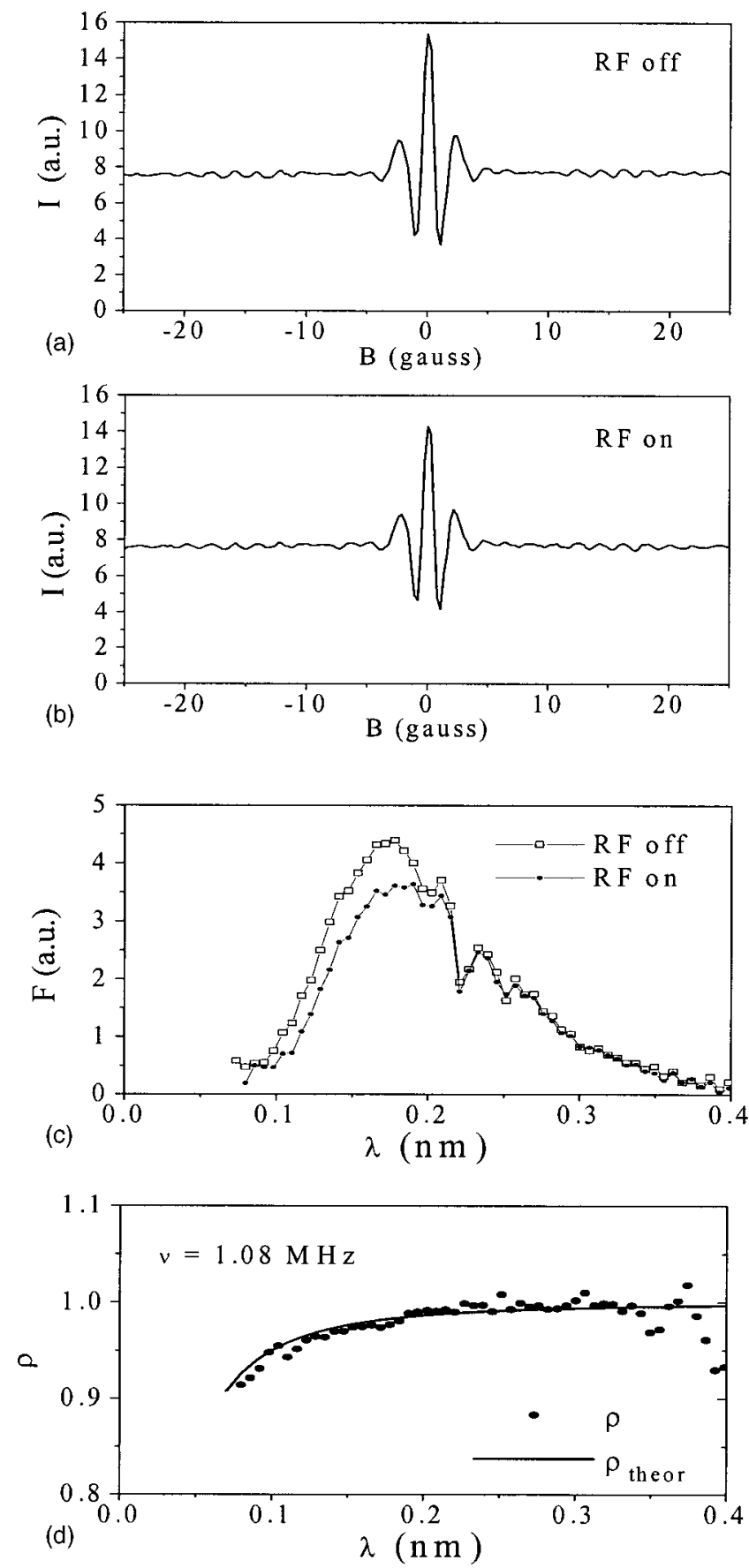

FIG. 5. Intensity of the white beam $I(B)$ as a function of the magnetic field $B$ : (a) when non-one-spin flipper is operating; (b) when all four flippers are switched on; (c) wavelength dependence of $F(\lambda)=s(\lambda) P(\lambda)$ obtained by Fourier transformation of the quantity $I(B)$ when non-one-spin flipper is operating (squares); and when all four flippers are switched on (circles); (d) the system adjusted at $\nu=1.08 \mathrm{MHz}$ : the spin-flip probability for one averaged flipper $\bar{\rho}$ as a function of wavelength (circles); the theoretical value of $\bar{\rho}$, according to Eq. (11), with averaged out sine square.

magnetic field. In the first experiment, the polarization as a function of the field $B_{1}$ in the Larmor coil (i.e., the SE signal) was measured for the white beam at several values of the permanent field $B_{0}$ in one flipper while the gradient $2 A$

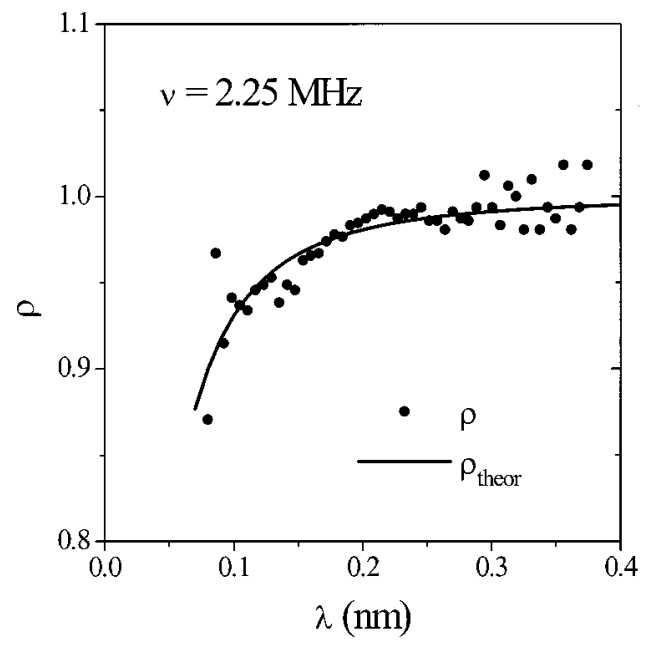

FIG. 6. The system adjusted at $\nu=2.25 \mathrm{MHz}$ : the spin-flip probability for one averaged flipper $\bar{\rho}$ as a function of wavelength (circles); the theoretical value of $\bar{\rho}$, according to Eq. (11), with averaged out $\sin ^{2}$ function.

of the field in this flipper remained constant and the parameters of the other flippers were also kept constant. Figure 8 shows the SE signals at three different values of $B_{0}$ when the rf coils are switched off. So these SE signals correspond to Larmor precession in the total field $B_{0}+A \cos (\pi x / l)$ of the flippers. It is observed that in order to compensate the additional phase $\Phi$ produced in one flipper by changing $B_{0}$, we need to increase $B_{1}$ as $B_{0}$ increases.

Figure 9 shows the same evaluation of the resonance signal as Fig. 8 when the rf coils in all flippers are switched on. In this case, the SE setup works in the NRSE mode. We can see that the position of the SE signal moves little as $B_{0}$ is changed. The amplitudes of the signals correspond to the initial polarization multiplied by $\rho_{1} \rho_{2} \rho_{3} \rho_{4}$, plotted in Fig. 4(a). The position of the SE signal as a function of the permanent magnetic field $B_{0}$ is presented in Fig. 10, when the rf coils are switched off (squares) and on (circles). When rf is off, the shift $\Delta \Phi_{\text {off }}$ of the position of the spin echo signal upon change of the permanent magnetic field $B_{0}$ is given by $\Delta \Phi_{\text {off }}=\int_{0}^{l}\left(\Delta B_{0}\right) d x$, hence linear in $\Delta B_{0}$. This is indeed seen in Fig. 10. When the rf is switched on, this change denoted $\Delta \Phi_{\text {on }}$ is given by Eq. (16). It is seen in Fig. 10 that the observed phase shift indeed agrees with the calculated one (solid line) taking $A=40 \mathrm{G}$. The main conclusion derived from this experiment is that the change of the permanent magnetic field in the flipper has little effect on the phase of the NRSE experiments.

Another manifestation of this effect appears in the comparison between the SE signals with rf coils switched on and off at $\nu=2.25 \mathrm{MHz}$ (Fig. 11). The strong permanent field, required by the resonance condition Eq. (4), produces a strong gradient of $B_{0}$ in the magnet over the cross section of the neutron beam. Different values of $B_{0}$ give different values of line integrals over the beam cross section. This results in decoherence over the beam, and the SE signal with rf switched off becomes smeared and hardly distinguishable from the noise [Fig. 11(a)]. When the rf coils are switched 

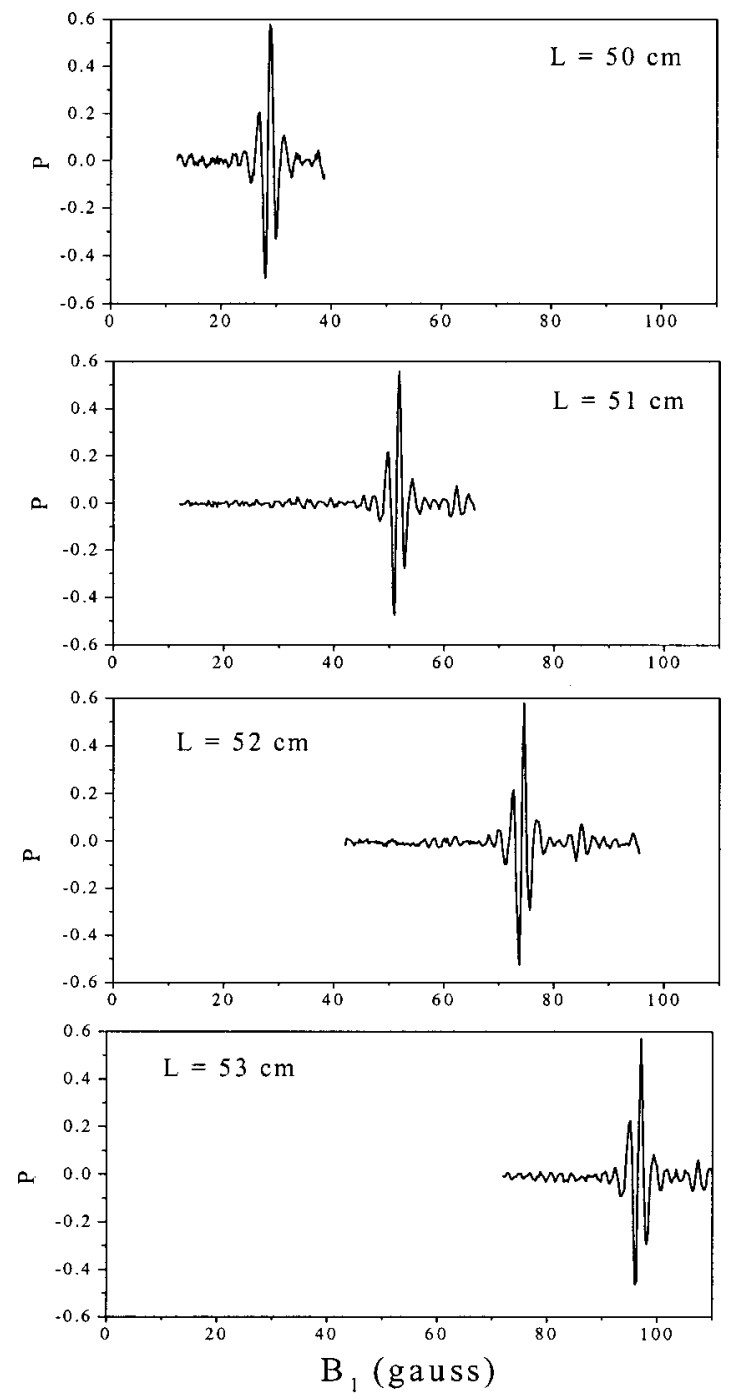

FIG. 7. Polarization $P_{y}$ : the spin echo signals at four different distances between first and second flippers $L$ $=50,51,52,53 \mathrm{~cm}$ at the frequency $\nu=1.08 \mathrm{MHz}$ as a function of the phase $\Phi$, i.e., as a function of the "compensating", field $B_{1}$ in the Larmor coil (LC in Fig. 3).

on, the phase shift is not so sensitive to variations of $B_{0}$, so the SE signal will be restored [Fig. 11(b)]. There is an additional source of stability of the phase when the rf coils are switched on. As was predicted in [18], the stability is also provided by the fact that the magnetic field on both sides of the magnets is mirrored and therefore variations of the permanent magnetic field will be compensated.

In the second experiment, we investigated the effects of the gradient $2 A$ of the magnetic field in one of the flippers on the phase shift at constant $B_{0}$. We measured the SE signals at several different values for the gradient $A$ in one flipper as a function of $B_{1}$, when the rf coils are switched off. Since the two halves of the gradient coil compensate their contributions to the phase $\left(\Delta \Phi_{\text {off }}=\int_{0}^{l}[A \cos (\pi x / l) d x=0]\right)$, the SE signal does not move with a change of the gradient. This is shown in Fig. 12 (open circles). When the rf is on, according to Eq. (16), the phase changes linearly with the absolute
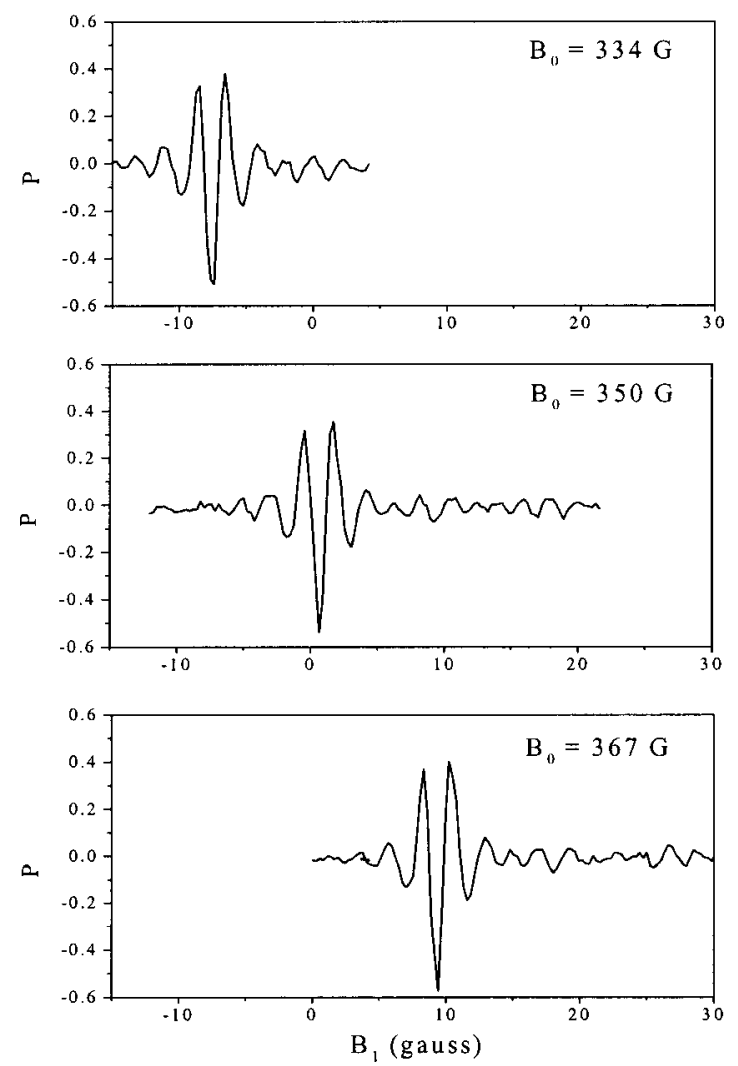

FIG. 8. Polarization $P_{y}$ : the spin echo signals at three different permanent magnetic fields $B_{0}=334,350,367 \mathrm{G}$ in one of the flippers as a function of the field $B_{1}$, when the rf coils are switched off.

value of the gradient. This was indeed found as shown in Fig. 12 (open squares). Results of the calculations with fixed $B_{0}$, while $A$ is varied, are also shown in Fig. 12 as solid lines. The theoretical curves and experimental points coincide.

\section{SUMMARY}

In this paper, we described the basic principles of neutron resonance spin echo using resonance coils with adiabatic passage of the neutron spin. The solution of the Schrödinger equation is derived for a specific configuration of the magnetic fields, required by adiabatic passage of the neutron spin in the resonance system. From this solution, we obtained the expressions for the probability of the spin-flip process as well as for the phase of the neutron wave, which the neutron experiences when it flies through the coil. It is observed from the theoretical expression as well as shown experimentally that NRSFs with adiabatic passage, in contrast to conventional resonance flippers, provide a neutron wavelength range of spin-flip high efficiency (probability $\rho=1$ ), limited only by a certain minimal wavelength. Since the spin-flip process with $\rho=1$ occurs for a broad wavelength spectrum, the whole spectrum of the neutron wavelength is involved in the precession in contrast to experiments with conventional resonance flippers.

The precession phase inside the coil is an addition of three contributions in the rotating frame approach. The first, big- 

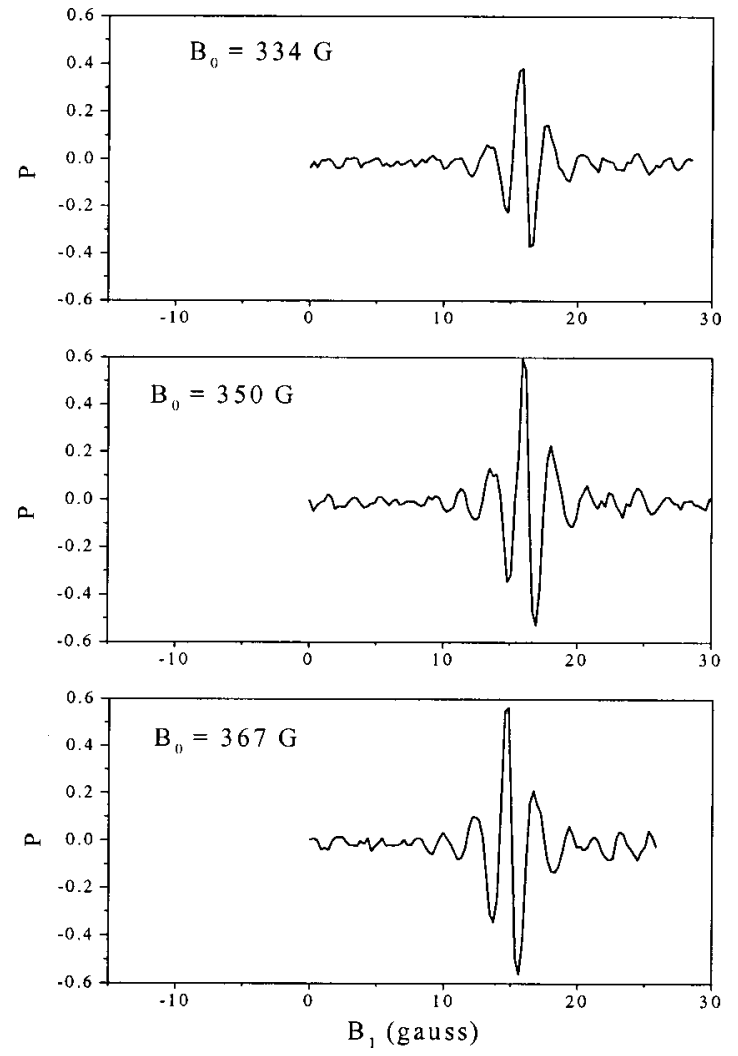

FIG. 9. Polarization $P_{y}$ : the spin echo signals at three different permanent magnetic fields $B_{0}=334,350,367 \mathrm{G}$ in one of the flippers as a function of the field $B_{1}$, when the rf coils are switched on.

gest contribution is the phase of the rotating frame $\omega_{0} \tau$. The second one is the precession phase of the neutron spin in the rotating frame since it follows adiabatically the effective field of this frame. The third, smallest one is Berry's phase due to the magnetic field that varies adiabatically in this

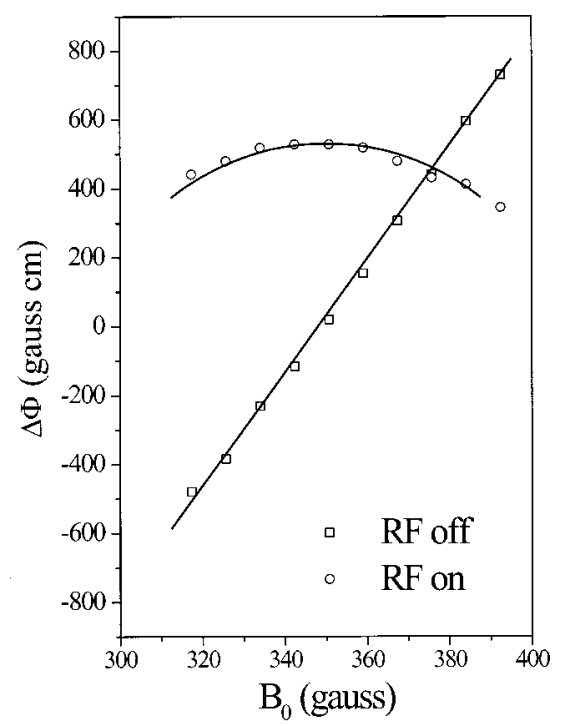

FIG. 10. Position of the SE signal as a function of the permanent magnetic field $B_{0}$ when the rf coils are switched off (squares) and on (circles). Results of the calculations are denoted by solid lines.
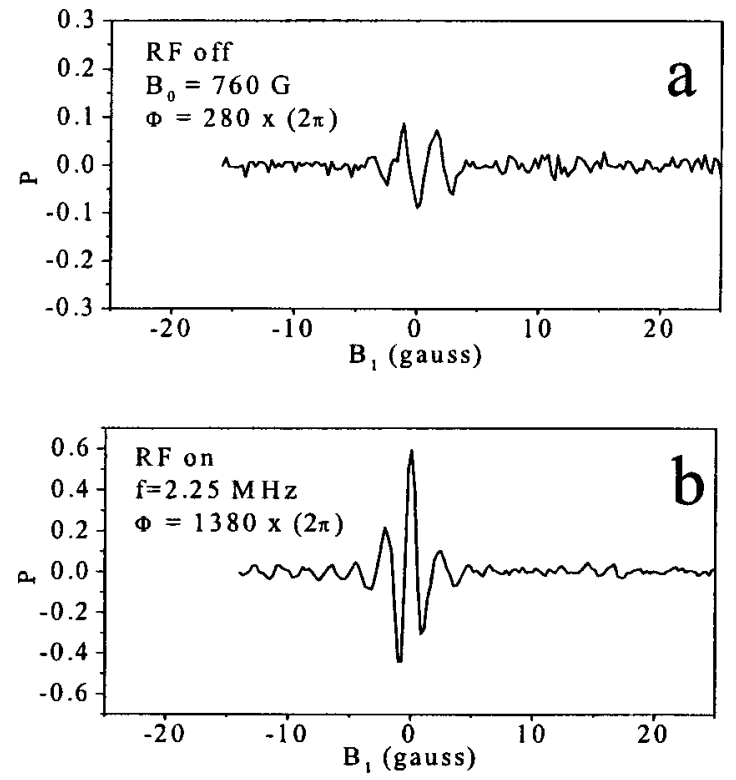

FIG. 11. Polarization $P_{y}$ : (a) the spin echo signals when the SE setup is adjusted for operating at $\nu=2.25 \mathrm{MHz}$ and rf coils are off; (b) the spin echo signals when the SE setup is adjusted for operating at $\nu=2.25 \mathrm{MHz}$ and rf coils are on.

rotating frame. Although we did not perform an experiment that would show the manifestation of Berry's phase, such an experiment is in preparation. The idea of this experiment is to measure the phase shift as a function of the magnitude of the magnetic field in the rotating frame for both negative and positive signs of the permanent-field gradient, using the principal scheme of the present installation. In full agreement with experiment, described in [12], we would observe the phase "jump" of $2 \pi$ in the range of the small values of the magnetic field. Since we work with the "white beam," we

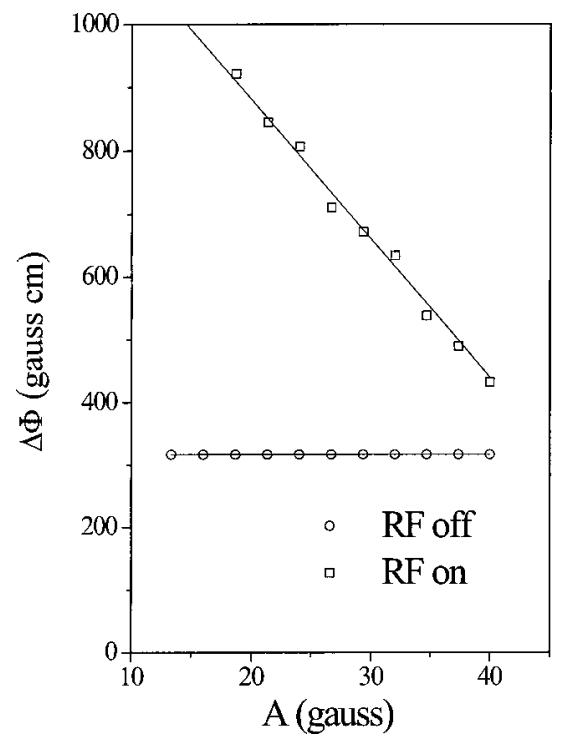

FIG. 12. Position of the SE signal as a function of the gradient of the magnetic field $A$ when the rf coils are switched off (squares) and rf coils are on (circles). Results of the calculations are presented on the figure by solid lines. 
can certainly identify the phase shift without ambiguity of multiples of $2 \pi$.

The phase shift between neutron spin states and the amplitude of the interference pattern are derived for the system of two coils and for the full SE setup, as is required for NRSE spectroscopy. In the experimental part of this work, we showed, in accordance with theoretical description, that the amplitude of the interference pattern for the white beam is proportional to the product of spin-flip probabilities of each coil $\rho_{1} \rho_{2} \rho_{3} \rho_{4}$. It was demonstrated that our setup simulated a dc field integral $2 \gamma B_{0} L$ with $B=365 \mathrm{G}$ and $B$ $=760 \mathrm{G}$ at $L=50 \mathrm{~cm}$ for the whole wavelength spectrum. It was also shown that the phase difference between interfering neutron waves is relatively insensitive to variations of the permanent magnetic field. Its stability is determined by variations of the gradient $2 \mathrm{~A}$, which is a much smaller quantity.

\section{ACKNOWLEDGMENTS}

The authors wish to thank J. Zandijk for developing and building the amplifiers to generate the rf fields. This work is part of the research program of the "Stichting voor Fundamenteel Onderzoek der Materie (FOM)," which is financially supported by the "Nederlandse Organisatie voor Wetenschappelijk Onderzoek (NWO)." The work was partly supported by the INTAS Foundation (Grant No. INTAS-9711329). One of the authors (S.V.G.) thanks RF FR (Project No. 00-15-96814) and the Russian State Programme "Neutron Research of the Condensed State."
[1] R. Golub and R. Gähler, Phys. Lett. A 123, 43 (1987).

[2] R. Gähler and R. Golub, J. Phys. (Paris) 49, 1195 (1988).

[3] R. Golub, R. Gähler and T. Keller, Am. J. Phys. 62, 779 (1994).

[4] F. Mezei, Z. Phys. 225, 146 (1972).

[5] S.V. Grigoriev, W.H. Kraan, F.M. Mulder, and M. Th. Rekveldt, Phys. Rev. A 62, 063601 (2000).

[6] F.M. Mulder, S.V. Grigoriev, W.H. Kraan, and M. Th. Rekveldt, Europhys. Lett. 51, 13 (2000).

[7] V.F. Ezhov, S.N. Ivanov, V.M. Lobashev, V.A. Nazarenko, G.D. Porsev, O.V. Serduk, A.P. Serebrov, and R.R. Tal'daev, Pis'ma Zh. Éksp. Teor. Fiz. 24, 39 (1976) [JETP Lett. 24, 34 (1976)].

[8] A.N. Bazhenov, V.M. Lobashev, A.N. Pirozhkov, and V.N. Slusar, Nucl. Instrum. Methods Phys. Res. A 332, 534 (1993).

[9] A. Abragam, Principles of Nuclear Magnetism (Clarendon Press, Oxford, 1961).

[10] S.V. Grigoriev, A.I. Okorokov, and V.V. Runov, Nucl. In- strum. Methods Phys. Res. A 384, 451 (1997).

[11] M.V. Berry, Proc. R. Soc. London, Ser. A 392, 45 (1984).

[12] T. Bitter and D. Dubbers, Phys. Rev. Lett. 59, 251 (1987).

[13] D.J. Richardson, A.I. Kilvington, K. Green, and S.K. Lamoreaux, Phys. Rev. Lett. 61, 2030 (1988).

[14] H. Weinfurter and G. Badurek, Phys. Rev. Lett. 64, 1318 (1990).

[15] J. Moody, A. Shapere, and F. Wilczek, Phys. Rev. Lett. 56, 893 (1986).

[16] R. Tycko, Phys. Rev. Lett. 58, 2281 (1987).

[17] D. Suter, K.T. Mueller, and A. Pines, Phys. Rev. Lett. 60, 1218 (1988).

[18] M. Th. Rekveldt and W.H. Kraan, J. Neutron Res. 8, 53 (1999).

[19] H. Rauch and S. A. Werner, Neutron Interferometry (Oxford University Press, Oxford, 2000).

[20] W.H. Kraan, J.B. van Tricht, and M. Th. Rekveldt, Nucl. Instrum. Methods Phys. Res. A 276, 521 (1989). 